\title{
The Changing Landscape for New Drug Development: Medical Countermeasures (MCMs) as a Case Study
}

\author{
Christopher-Paul Milne
}

\subsection{Introduction}

The prospects of a new medicine making it from the laboratory bench to the pharmacy shelf are daunting. For each drug that makes it into the clinic for testing in humans, thousands do not, for myriad reasons ranging from easy-to-understand ones such as toxicity in animals to more esoteric ones such as "druggability" (i.e., simply put, the likelihood of being able to modulate a target with a drug). Once in the clinic, the odds are a little better that a lead compound will emerge as the target product of a new drug application or biologics' license application and be approved by National Regulatory Agencies (NRAs) - those odds are typically calculated to be about 1 in 10 . Still this is not good news for drug sponsors, who may spend as long as two decades and over $\$ 1$ billion USD out-of-pocket to get a new drug to market. But over the last decade, the news has become even more daunting. Risks associated with drug and biologics research and development (R\&D) have only grown over time, while rewards are diminishing. Average time before

C.-P. Milne ( $\square)$

Tufts University School of Medicine, Boston, USA e-mail: sirenlim@gmail.com

C.-P. Milne

University of Edinburgh, Edinburgh, Scotland, UK follow-on competitors chase a first-in-class drug into the marketplace shrink each decade by several fold, and the percentage of prescriptions filled by generics doubles decade by decade (now reaching as high as $90 \%$ in many developed country markets). More telling for the demise of the traditional blockbuster strategy for achieving a sustainable return-on-interest (ROI) is that average sales for new launches were lower by $40 \%$ in 2010 from what they were just 5 years earlier [1] and declined to $2 \%$ from the $10 \%$ achieved in 2010 as of 2018 [2]. This new reality is often heralded as a healthy development for healthcare cost containment, even though drugs typically comprise a relatively minor share of the total bill for health care, for example, $10 \%$ in the USA [3]. However, for those concerned with not only controlling the escalation of healthcare costs but also curtailing health risks, these circumstances represent a different set of perils. Drugs that present too difficult a development challenge or too uncertain a market will not be able to compete successfully for resource allocation internally within companies or externally in the capital marketplace. These perils are particularly acute in new fields of product development like regenerative medicine or even a new sub-sector derived from several existing fields of product R\&D - such as Medical Counter Measures (MCMs), which are products that may be used in the event of a potential public health emergency stemming from a terrorist attack with chemical, biological, or radiological/nuclear (CBRN) 
agents or a naturally occurring, emerging or re-emerging infectious disease.

The product sector case example for this overview of the shifting landscape for new drug development (including both small molecule and large molecule drugs, respectively of chemical or biological origin) will be MCMs, and the regional focus will be primarily on the USA. Fully half of new active substances (NASs) approved globally each year, ownership of the extant R\&D pipeline worldwide (for MCMs and in general), as well as the end-market for all medicines occur within the purview of the USA. So as the USA goes, so goes biopharma world. For example, historically most of the regulatory innovations of the twentieth century, such as the Orphan Drug and Fast Track programs, were first introduced in the USA and soon emulated by Europe and Japan, while in the twenty-first century, a number of emerging market countries (especially those in the Asia-Pacific region) base their regulatory and reimbursement evaluations on those conducted in the USA, especially for breakthrough products, for which the resources and expertise for developing the evidence base to make such decisions (e.g., regulatory science tools and/or real-world data) are limited in emerging market countries. In addition, the US National Institutes of Health (NIH) and the U.S. Food and Drug Administration (FDA) lead their respective fields of research and regulation due to the number and expertise of their staff, the size of their budgets, as well as global influence and interest.

The remainder of this chapter is comprised of materials adapted from previous publications authored by the Center for the Study of Drug Development at Tufts University School of Medicine (Tufts CSDD) over a ten-year period from 2010 through 2019. In addition, there are occasional infusions of updated commentary to "connect the dots" of how we got to where we are today. These publications may be requested from Tufts CSDD (if originally published in-house) or through the usual channels for requesting articles published in the public domain (permission to reprint the articles having been granted, where required). The text of the chapter is structured basically in a chronological fashion beginning with Tufts CSDD analysis of the early era of MCM evolution as a sub-sector from various extant therapeutic areas. It then chronicles the changes to the $R \& D$ paradigm in response to the challenges that emerged for both MCMs and biopharma in general. Finally, it ends with an exploration of the devolution of the MCM sub-sector back into its roots in the infectious disease area as an increase in actual outbreaks as well as other signals of global vulnerability to pandemic threats have minimized the MCM emphasis on biodefense against a wide range of CBRN agents in favor of public health tactics to address humankind's maladaptation to a world in which it is constantly assailed by its microbial competitors and symbionts, or to novel public health crises of its own making.

\subsection{Early Evolution of a New R\&D Sub-sector [4]}

Historical Background-Historically, in the USA, even though the "war on cancer" had been declared in the early 1970s, awareness of the chasm between risk and reward in biopharmaceutical R\&D first became a political issue in the 1980s, initially through advocacy efforts on behalf of victims of rare diseases. In aggregate, rare diseases comprise a large "special patient" population of 20-30 million each in the USA and European Union and perhaps as many as 400 million worldwide, but this population is spread over approximately 7000 small disease markets, and so became therapeutic "orphans" due to the lack of incentives for product developers willing to foster R\&D programs for these rare conditions. Close on the heels of the economic epiphany revealed by the orphaning of rare disease due to business reasons, the AIDS epidemic struck. In true epidemic fashion, AIDS quickly fomented a crisis for a healthcare system in the USA that had no weapons with which to stem the bewildering tide of morbidity and mortality, whereas cancer and HIV were rampant killers in the 1980s and 1990s, and budget thieves for healthcare systems as well; today scores of new medicines have blunted some of their public 
health impact and often have commuted imminent death sentences to chronic disability - with variable levels of quality of life, but life nonetheless. Other diseases have now emerged to take their place; some, such as antibiotic-resistant infections, are as lethally inexorable as AIDS once was. Others, such as depression and obesity, kill more slowly but in great numbers across the spectrum of age ranges, just as cancer once did, unchecked and with little medical recourse. The difference over the years was that AIDS, rare diseases, and cancer developed a vocal and organized advocacy that affected political change, which in turn laid the statutory groundwork on which the USFDA built the designation programs to address the unmet medical needs of these special patient populations.

In the early 2000s, a frightening new public health reality dawned, as it became clear in the wake of $9 / 11$ that terrorist attacks are a very real threat that could cause a large number of civilian casualties. Incidents involving anthrax, a commonly occurring bacterium, and sarin, a chemical toxin from a commonly used family of pesticides, demonstrated the potential for bioterrorists to use a wide range of CBRN agents in future attacks. Awareness of naturally occurring pandemics has also been increasing. The range of infectious threats include: emerging infectious diseases like severe acute respiratory syndrome (SARS) and avian flu (H5N1); re-emerging diseases like measles, pertussis, and Ebola; "neglected diseases" like tuberculosis, dengue, and water-borne parasites; and bioterror agents such as smallpox and anthrax. SARS was the first severe newly emergent infections of the twenty-first century and also demonstrated two significant characteristics of the new plagues: The first is their potential for significant direct (i.e., medical and government program costs) and indirect economic impacts (e.g., Asia-Pacific economy lost nearly $\$ 40$ billion); and second, even relatively quick-kill infections can spread rapidly due to modern travel and the global nature of business (i.e., approximately 250 cases of SARS in 10 countries spread within a few days from a professor, who had been treating SARS patients in the Chinese countryside and then traveled to a popular hotel in Hong Kong).

In response to these threats, it became imperative that a biodefense system of pandemic and bioterror medical countermeasures (collectively, MCMs) had to be developed. Many of the identified threats, even from pathogens known to be highly lethal and transmissible, did not have optimal or, even sometimes, any treatment options at all, beyond supportive care. At this time, however, the research and development of MCMs was typically viewed as "stagnant or non-existent" among major biopharma firms. As a consequence, what little $R \& D$ that took place was at small companies funded by venture capital with little institutional memory for managing a product from discovery through licensing to the marketplace. To this end, the US government (USG) passed a series of laws to stimulate countermeasure development that encompassed programs and funding for basic research, advanced development of technology, and acquisition of product for stockpiling in emergencies or prophylactic use by the military and first responders.

Roles of the Public and Private Sectors-The shape-shifting of the biodefense industry in the USA began in 2002, with the Bioterror Act, which called for development and monitoring of a stockpile of bioterror countermeasures. Several years later, BioShield I was passed in order to create a market for MCMs by setting up a special reserve fund for the purchase of medical countermeasures to be stored in the strategic national stockpile (SNS) and available for quick distribution in the event of an attack. This was soon followed by the Pandemic and All-Hazards Preparedness Act (also known as BioShield II), which brought both pandemic and bioterrorism legislation into one bill with the intent of generating incentives for entry into the business of biodefense. BioShield II established the Biomedical Advanced Research and Development Authority (BARDA) within the federal department of Health and Human Services (HHS). BARDA is the central authority in countermeasure development and administers the Biodefense Medical Countermeasure Development Fund, 
which expands the options for procurement funding to include milestone payments, awarding exclusive supplier status, establishment of domestic manufacturing capacity, and dosing and administration studies.

Yet even by FY2010, the entire biodefense allocation comprised only $0.1-1 \%$ of the budgets for the main USG departments responsible for protecting the public - Health \& Human Services (HHS) - which includes FDA, the Department of Homeland Security (DHS), and the Department of Defense (DoD). Biotech and pharmaceutical companies read the "tea leaves" of these relatively small numbers as indicating that the US Congress was not serious about investing in the development of medicines and vaccines against bioterror threats.

But the USG did want to encourage more interest from the private sector but was at odds how to do it. Consequently, it took only tentative baby steps in that direction. HHS proposed pilot studies to promote a more synergistic working relationship among government scientists at $\mathrm{NIH}, \mathrm{CDC}, \mathrm{FDA}$, and BARDA. Predictably, the response from industry and investors was ho-hum. Guidance on cell-based vaccine production was finalized by FDA, but during drafting failed to elicit even a single industry comment despite being "advertised" in an HHS press release. With a few exceptions, there was almost no interest by private investment firms and funds in biodefense due to its perception as defense contracting with lower margins, smaller markets, and one-off product sales. The basic problem was laid out by Chuck Ludlam, former top staffer for BIO and Senator Joe Lieberman (I-Conn) at an Institute of Medicine (IOM) meeting in $2010^{1}$ :

$$
\begin{aligned}
& \text {...firms need "goal line" incentives such as "cash, } \\
& \text { tax benefits, patent benefits and liability } \\
& \text { protections." }
\end{aligned}
$$

Near the end of the 2000s, the private sector MCM pipeline was comprised by somewhat over a hundred, but less than two hundred companies, mostly start-ups and small/medium-sized

\footnotetext{
${ }^{1}$ As reported in the Pink Sheet (Informa subscription newsletter) on April 12, 2010 at 24.
}

enterprises (SMEs). As seen in Fig. 7.1, for Big Pharma companies with any MCMs in their pipelines, the total number of products in development approached 1000, but well short of $10 \%$ could be categorized as MCMs. Among start-ups and SMEs combined, there were approximately an equal number to Big Pharma of products in the R\&D pipeline overall, but $42 \%$ and $25 \%$ respectively were MCMs. Although the majority of start-up and SME MCM pipelines were at Phase 1 or earlier, Big Pharma had a considerable number in later stage development (over 40\%). Thus, although Big Pharma players in the MCM field were small in number, they were large on impact. However, even early in the evolution of the MCM sector, its fortunes as a whole appeared to be more likely to wax and wane in sync with the fortunes of the start-ups and SMEs based on their greater dependence on and resource commitment to MCMs. The paramount question that loomed in the background was whether MCMs would emerge as a new business sector under a traditional pharmaceutical business model or evolve into something similar to what was at the time still a shape-shifting biotech business model?

Business Models: Old Paradigm-Under the traditional market model, there were two basic strategies for MCMs: the "One drug, one bug," or so-called "fixed defenses" approach versus the development of multi-purpose countermeasures, so-called "flexible defenses," such as immunomodulators, better delivery systems, or prototype vaccines that can be easily tailored to emerging noxious agents. The flexible defense strategy is more functional given the unpredictable nature of the threat but also more economically attractive because flexible broadspectrum products will have markets beyond government purchases.

The MCM product range consisted of: vaccines, therapeutics, diagnostics, immunomodulators, platform technologies (e.g., including some current or back-burner technologies such as new methods of drug delivery, specialized enzymes for decontamination, technologies for faster design and production of vaccines or antibodies against new strains or new microbes and 


\begin{tabular}{|l|c|c|c|}
\hline & Big Pharma & SMEs & Start-Ups \\
\hline $\begin{array}{l}\text { Total number of } \\
\text { products in pipeline }\end{array}$ & 833 & 591 & 104 \\
\hline $\begin{array}{l}\text { Percent of products } \\
\text { that are MCMs }\end{array}$ & $6.8 \%$ & $25.2 \%$ & $42.3 \%$ \\
\hline $\begin{array}{l}\text { Percent of products } \\
\text { in phase 1 or earlier }\end{array}$ & $56.1 \%$ & $89.3 \%$ & $100 \%$ \\
\hline
\end{tabular}

Fig. 7.1 MCM pipeline snapshot in 2008

products that create molecular barriers to infection at vulnerable sites like mucous membranes), as well as antidotes, chemoprotective agents, ancillary treatments, or prophylactic measures to mitigate, prevent or treat illness resulting from intentional bioterrorist attacks with CBRN agents or naturally occurring pandemic disease.

Traditional Business Models- "Biotech Business Models," an article by L. Paveras, ${ }^{2}$ discusses the business models being utilized at the time for biotech R\&D:

(1) FIPCO (fully integrated pharmaceutical company) — brings a product to market after early identification of lead or acquisition of promising compound;

(2) RIPCO (royalty income pharmaceutical company) identifies and takes lead compound through proof-of-concept, then sells off or partners with other firm, usually Big Pharma/Biotech;

(3) Technology Platform-develops new technology and creates specialty line of products or exploits it through licensing, partnering, etc.

(4) NRDO - "no research, development only"firms that usually acquire or license-in leads that Big Pharma/Biotech is not interested in commercializing in-house;

(5) Product development partnerships (PDPs) or private-public partnerships (PPPs);

(6) Virtual R\&D (e.g., Battelle)—U.S. Department of Defense (DoD) preferred providers who sub-contract out.

\footnotetext{
${ }^{2}$ See Paveras, L. "Biotech Business Models," http://www. healthonomics.org/2008/01/biotech-business-models.html.
}

The major operational features of the traditional models were government push and pull incentives, mainly the USG (but also the EU Innovative Medicines Initiative-IMI) as well as risk-sharing opportunities through Cooperative Research \& Development Agreements (CRADAs in the USA) and consortia. Much of the fundamental impetus from push incentives derive from increased funding for basic research to develop intellectual property (IP) that can be transferred to private sector companies. Other push incentives were cost-sparing measures such as liability protection under vaccine compensation laws as well as tax credits.

While government expedited development and review programs can get a product to market quicker (i.e., push incentive) and market protection awards can keep it on the market longer (i.e., pull incentive), for MCMs the most significant of the pull incentives was procurement contracts. Procurement contracts guarantee companies that countermeasures developed from promising candidates will be purchased by government agencies. Other incentives for companies to enter biodefense apply mostly to SMEs, as explained by AVI Biopharma President and COO Alan Timmins $^{3}$ :
"While we're working with the government on some specific viruses that are very lethal, we're learning a lot about our own technology: how to apply it, where it works best, where it might not work so well," said Timmins. "So we get a lot of benefit at no cost to us. And we get enhanced credibility within the marketplace for drugs and

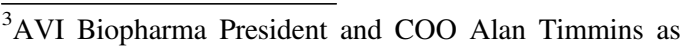
quoted in the trade magazine Bioexecutive International (April 2007 at 22-27).
} 
within the stock market because there's cachet in having the government as a partner."

In related fashion, government incentives are sufficient to stimulate interest from biotech companies, for whom an inflow of tens or hundreds of millions of dollars will have a significant impact. Nevertheless, Senator Lieberman explains in his testimony before a US Congressional Committee that there are other "benefits" to USG funding as well ${ }^{4}$ :

The only companies that are likely to accept a defense contractor model are companies with no approved products, no revenue from product sales, and no other source of capital to keep the lights on. For them government funding is "non-dilution" capital, meaning it's a form of capital that does not dilute the ownership shares of its current shareholders. Many biotech companies have stock trading in the low single digits, so they cannot issue another round of stock that would enrage the current shareholders. For them this government funding might validate the scientific platform of the company, generate some revenue, and hype the stock.

As a market, MCMs have many weaknesses. For example, antimicrobials have a shorter product life cycle due to the development of resistance. Vaccines are typically administered to healthy people and so have a higher risk-benefit threshold, and thus greater litigation exposure. Overall, MCMs are typically not treatments for chronic diseases, which offer a repeat sale market. In addition, generally speaking there is dependence on a single customer, often a government agency, and as with all large volume purchasers, margins are lower. Also, the government can be a difficult and sometimes unreliable business partner. For example, there was the well-publicized Cipro incident when the government was seen as extorting a low ball price, while another major problem is cancellations of request for proposals (RFPs).

In order to expand the government market and attract additional institutional purchasers, developers must optimize product characteristics such as: durable storage properties, convenient "kit"

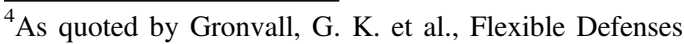
Roundtable Meeting, http://igcc.ucsd.edu/pdf/1ALieberman TestimonyOctober6.pdf.
}

packaging with attached patient/prescriber information and supplies for administration, minimal need for boosters, as well as a manageable expiration and replacement cycle. Getting products approved and into the medical armamentarium does not mean that the challenges are over. In January 2003, the Bush Administration had a goal of immunizing 500,000 health workers for smallpox within 30 days and 10 million emergency response personnel within a year, but five years later only 40,000 were actually vaccinated due to problems with unexpected side effects, worker compensation issues, and liability concerns. Of the 95 patients who contracted measles during the 2008 outbreak in the USA and were eligible for vaccination, 63 were unvaccinated because of their parents' philosophical or religious beliefs.

Business Models: New Paradigm-In his 2006 text on the future of biotech, Harvard Business School professor and economist Gary Pisano describes various types of technological innovation (e.g., novel research methods and tools, novel targets or mechanisms and novel compound types/treatment modalities/markets), and the factors that companies with different types of innovation must consider when planning a business model. ${ }^{5}$ Pisano explains that there are four factors that determine whether "a market for know-how" will succeed or fail: information asymmetry; specialized assets; tacit (not easily transferable) knowledge; and intellectual property (IP) protection. Continuing IP legal battles aside, when markets for know-how work, business models that involve out-licensing technology increase efficiency and can create handsome returns. When markets for know-how fail, business models with vertical integration may be the most effective strategy, if enough capital is available and attainable. Is there a market for know-how in MCMs?

The MCM sector is built on new technology platforms to identify novel pathogens in the

\footnotetext{
${ }^{5}$ Pisano, G. P. Science Business: The Promise, the Reality, and the Future of Biotech; Harvard Business Press, Boston, MA, USA. November 2006; ISBN 13:978 1591398400.
} 
laboratory and in the field, discover novel target pathways to neutralize or prevent illness as well as to improve existing ones, and apply this knowledge to the development and production of MCMs, i.e., a market for know-how. This is a new market for know-how different from the one in which Big Pharma is the acknowledged leader, like cardiovascular or GI disease markets, where numerous drugs have been developed and an infrastructure is in place. Most government grants for civilian biodefense awarded to the private sector have gone to biotechs. This has engendered something akin to a working relationship between government and the biotech sector, albeit more like a mutual dependency. However imperfect, it is a better relationship and thus more valuable than what had previously existed between Big Pharma and governments. In addition, most biotechs in the biodefense field have specific platform technologies and research expertise that extends farther upstream in the $R \& D$ continuum than the clinical focus of most Big Pharma. This results in both information asymmetry and a disparity of tacit knowledge between biotech firms and Big Pharma (a potential competitor and/or buyer) on the one hand, and between biotech and government (a potential buyer) on the other.

Some of the work in MCMs could also be considered specialized assets. These are assets that are not easily applied to alternative uses. Once invested in a specialized asset, it is very difficult to switch gears. The market for know-how generally does not highly value the prospects of being locked into an investment for specialized and limited applications. Indeed, while some companies with a biodefense core focus are developing very specialized technology, many are working with multi-purpose platforms applicable to various therapeutic areas and are able to spread risk through collaborations (now increasingly with Big Pharma and Big Biotech).

If there is a functioning market for know-how with the government as the buyer as well as Big Pharma, but the market is limited, at least as far as the biodefense MCM space is concerned, there is a role for integrated business models, such as
FIPCO, that SMEs with a core MCM focus may utilize to reach their goals. The MCM market is complicated by the influence of the government intervention in the market dynamics by supporting a market for know-how to a limited extent as well as being a source of capital for SMEs that adopt a vertical integration approach, but again to a limited extent. Since the market for know-how is limited to a cadre of SMEs (and start-ups that evolve into emerging SMEs) with a core focus on MCMs (mostly vaccines and technology platforms), this leaves room for other business models and sector players to address the remaining market needs. For fixed defenses, such as MCMs for some CBRN and known pandemic threats (e.g., antibiotics, antivirals, and treatments for acute radiation sickness (ARS) and chemical poisons), it would be Big Pharma, employing a FIPCO or NRDO model, using incremental innovation within their existing portfolios. For diagnostics, specialty pharma or biotechs are the likely players using FIPCO, or RIPCO involving partnering or out-licensing to Big Pharma/Biotech. For flu vaccines, it would be Big Pharma/Biotech and specialty pharma/biotech, perhaps in partnership. In addition, there is a significant role to be played by the government in both fixed and flexible defenses. For fixed defenses, the government's role would be as purchaser, and a lesser role as provider of $\mathrm{R} \& \mathrm{D}$ funding, and a limited role as the player of last resort in product development and manufacture. For flexible defenses, the government would have to play all three roles in order to generate an effective capacity.

By the end of the 2000s, a US presidential commission of influential leaders and experts came to appreciate three crucial realities. ${ }^{6}$ The first is that "... efforts to address biodefense and emerging infections are mutually supportive and that compartmentalizing these efforts is arbitrary and counterproductive." The second is an extension of the first, i.e., the recognition that the ends and thus the means must go beyond even pandemics and WMDs (i.e., weapons of mass

\footnotetext{
${ }^{6}$ As reported in National Health Security Strategy for the U.S., Objective 6, December 2009, at 13-14.
} 
destruction). Among HHS recommendations for action were that investments should focus on new technologies or MCMs that could also have uses in non-public health emergency situations, and should address the continuum from research to delivery. Investments should be prioritized to effectively pursue those countermeasures that have the greatest potential to improve national health security, prevent or limit the spread of disease, limit the clinical impact of a health incident, and have elements with potential widespread application even in the absence of a catastrophic event. Third, somebody has to take charge.

Update and Commentary-Looking back in 2018, some of what these government and industry experts proposed actually happened and improved the overall funding environment for MCMs, if not the actual performance of the sector. President Obama implemented a strategy that combined efforts aimed at addressing both deliberate and accidental threats to US (and global) public health and BARDA more or less became the go-to agency for this initiative. While it is unclear what exactly was expected in terms of somebody taking charge, start-ups and SME biotech companies were recognized as the most promising candidates to foster development of new medical countermeasures, and the USG began to focus its funding and assistance on these companies. They face serious challenges related to manufacturing capacity and negotiating the regulatory labyrinth, according to Battelle's Senior Market Manager for Medical and CBRN Products Russell Coleman. Examples of initiatives in place to address these challenges include: Advanced Development and Manufacturing capabilities (ADMc), which provides both development and manufacturing resources to smaller biotech companies as needed; and the Medical CBRN Defense Consortium (MCDC), an organization that aims to help smaller companies who wish to work with the DoD navigate the process of becoming an approved federal contractor [5].

Meanwhile, even as Big Government's attitude toward SMEs began to change, Big Pharma was undergoing its own change of direction, becoming more willing to consider specialty markets and increasingly investing in historically unattractive markets like orphan drug development and vaccines, and subsequently in MCMs. Industry formed consortia with the public sector to address some of the R\&D problems inherent in the field, exemplified by the work of the Alliance for Biosecurity (formed in 2005, consisting of approximately 15 member companies to foster private-public partnerships for MCM development) such as the development of appropriate animal models. In contrast to the field of countermeasures for emerging and little known pandemic threats, populated mostly by biotech start-ups and SMEs, the market for known pandemic countermeasures, i.e., for various forms of flu, has been so far supplied primarily by Big Biopharma. And business is good! The estimated global flu market for therapeutics and vaccines (20\% and $80 \%$ of the market respectively) is estimated to be worth $\$ 10.2$ Billion by 2022 [6].

\subsection{Impact Factors for Sector Building in the Twenty-Teens [7]}

\subsubsection{Facilitated Regulatory Pathways (FRPs)}

Broadly speaking, special designation programs such as those implemented by the USFDA-orphan, priority review, accelerated approval, fast track, breakthrough therapy designation (BTD) - have been to expedite and sustain development and facilitate authorization of new medicines for unmet medical needs through so-called pushpull incentives. Although generally successful over time, their success has been confined to certain therapeutic areas and, within those areas, certain diseases. Times have changed. The research and development (R\&D) burdens and public health urgency that acted as an impetus for the FDA to intervene more actively for certain disease areas are now broadly experienced across many disease areas. This betokens the need for the FDA to make designation and implementation decisions with a view that reaches beyond 
the immediate horizons of political expediency and patient advocacy to encompass the broader expanse of factors that now influence $R \& D$ decisions-public and private sector prioritization, new players in the paradigm, and patient-focused drug development.

Orphan Designation-Among the FRPs, the Orphan Drug Act was the first "push-pull" incentive (early 1980s in the USA, 1990s in Japan, and 2000s in the EU) and has been arguably the most successful. The push incentives lower the logistical and financial barriers for entry into the field of $R \& D$ for rare diseases (i.e., those with a prevalence of 200,000 patients or less, or unlikely to recoup $R \& D$ costs from market returns) and include waiver of user fees, technical and administrative assistance by the FDA's Office of Orphan Products, and clinical research grants. Pull incentives increase the likelihood that if the products reach the market, there will be sufficient return on investment. Pull incentives encompass tax credits for as much as $50 \%$ of clinical development costs and, most importantly, the so-called orphan exclusivity that prohibits the FDA from approving a marketing application for the same drug that treats the same condition or illness for 7 years from the date of approval of the first orphan application, even in the absence of a patent.

If the measure of success for FDA special designation programs was the orphan drug program, one would have to say that they have worked very well. Some form of the program has been adopted worldwide in the major geographic loci of medicines $\mathrm{R} \& \mathrm{D}$, and elements of the push-pull approach have informed incentive programs for other unmet needs right up until the present time. The program broke new ground before the ground was even recognized on a number of fronts: patient-focused drug development, targeting subsets of diseases, and proving that there was a viable economic model in small markets-giving birth to the term of niche blockbusters (i.e., the number of orphan drugs in the top 200 for US sales increased fourfold over the 2000s).

Accelerated Approval was another early special program in the USA for expedited development. Accelerated approval regulations were promulgated on December 11, 1992. The law stipulates that drugs must be intended for patients with serious illnesses. Moreover, the data used for the accelerated approval must show an independently corroborated effect on an as yet unvalidated surrogate end point that is reasonably thought to be predictive of clinical benefit. Upon completion of Phase 4 trials that confirm a clinical benefit (i.e., that the "new" surrogate was indeed predictive of clinical benefit), a traditional full approval may be awarded. If the confirmatory trial does not show that the drug provides clinical benefit for patients, the FDA has regulatory procedures in place that could lead to removing the drug from the market. If a company seeks accelerated approval based on restricted distribution, then it must have clear distribution restriction practices and provider/user education programs for the drugs to gain approval.

Priority Review introduced the concept of a premium for novelty. In 1992, the FDA agreed to specific goals for improving drug review time by creating a two-tiered system of review times: standard and priority reviews. Standard review is applied to a drug that offers, at most, only minor improvement over existing marketed therapies. A priority review designation is given to drugs that offer major advances in treatment or provide a treatment for which no adequate therapy exists. Designation of a drug as "priority" alters neither the scientific/medical standard for approval nor the quality of evidence necessary but simply the amount of time (6 months) that FDA has to review (i.e., take first action: accept, reject, needs more work) the marketing application as opposed to 10 months for an application given a "standard" review. ${ }^{7}$ The distinction between priority and standard review times is that additional FDA attention and resources will be directed to drugs that have the potential to provide significant advances in treatment, prevention, or diagnosis,

\footnotetext{
${ }^{7}$ In the mid-2000s a two-month filing review period was added to priority and standard review time goals.
} 
including expansion of indications to a new subpopulation, such as children. ${ }^{8}$

Fast Track Designation - can be requested by the drug sponsor at any time during development. It allows sponsors to increase their scientific interaction with the FDA through more frequent meetings and written correspondence, as well as to submit completed sections of the new drug application to the FDA for "rolling review" rather than having to wait until the entire application is complete. In addition, as the FDA notes on its Web site, most drugs eligible for fast track designation are likely to be considered appropriate to receive a priority review. Therefore, the designation can act as a push incentive by expediting the development and review process, lowering the cost burden upfront for bringing a product to market. It can also act as a pull incentive by providing the product earlier access to the market, thus allowing more of the patent life to run while the product is actually on the market earning returns rather than during the development period when investment funds are being "burned" without replenishment from sales. Fast track was the most significant factor in a recent study of 20 variables affecting the likelihood of first-cycle approvals, with $78 \%$ of fast tracks achieving this milestone. Investment boost seems to be a significant result of being awarded a fast track designation - an analysis by a consulting firm showed an $18 \%$ increase in stock valuation on the first day after designation announcement. A study by the Tufts Center for the Study of Drug Development confirmed this effect by demonstrating a statistically significant percentage of change in stock price $(P=0.03)$ and upward difference in stock price $(P=0.04)$ after designation was publicized.

\footnotetext{
${ }^{8}$ Subsequently, priority review was expanded into the Priority Review Voucher (PRV) program adopted to incentivize drug sponsors by awarding an obligation on the part of the USFDA to consider a drug for priority review if its sponsor had received a product approval for another disease indication listed as important to global health (in three categories: neglected tropical diseases, rare pediatric diseases, or medical countermeasures). How much of an incentive it is remains to be seen, especially for medical countermeasures? Although MCMs were added to PRV program in 2016 under the twenty-first century Cures Act, as of the end of FY 2018 only one PRV has been awarded to Siga Technologies.
}

Breakthrough Therapy Designation (BTD) The FDA Safety and Innovation Act of 2012 (FDASIA) was the congressional response to stakeholders' calls for an upgrade and update to FDA special designation programs. FDASIA changes focused on accelerated approval in particular and, to a lesser degree, on priority review and fast track, as well as adding a new program, the Breakthrough Therapy Designation (BTD). The FDA's response in turn was the 2014 publication of a draft guidance on expedited programs for serious conditions. In the guidance, the scopes of priority review and fast track were expanded to include qualified infectious disease products (QIDP) as directed under the Generating Antibiotic Incentives Now (GAIN) Act. ${ }^{9}$

The guidance also broadens the use of accelerated approval to "cases in which the advantage of a new drug over available therapy may not be a direct therapeutic advantage, but clinically important improvement from a patient and public health perspective." At the same time, the guidance broadens the scope of the empirical evidence of clinical benefit from surrogate end points, or intermediate clinical end points (e.g., ones reasonably likely to predict an effect on irreversible morbidity and mortality), to include evidence that may be provided by such evolving technology as biomarkers and "other scientific methods or tools." Like fast track and accelerated approval, the first level of eligibility for the BTD is that the drug (or biological drug product) treats or intends to treat a serious condition. BTD distinguishes itself somewhat in the second eligibility criteria by requiring clinical evidence as the quantum of proof, as opposed to fast track, for instance, in which non-clinical data (or clinical data) may be used. Similarly, the third arm of eligibility for the BTD is that the drug demonstrates substantial superiority over available therapies on a clinically significant end point, whereas fast track requires only a demonstration of the potential to address unmet medical need and accelerated approval requires that it provides

\footnotetext{
${ }^{9}$ With dramatic results-74 QIDP designations awarded in the first 5 years of the program according to the General Accounting Office (GAO) of the US Congress.
} 
both meaningful advantages over available therapies and evidence that reasonably predicts clinical benefits. The benefits of the BTD are more expansive than fast track and in fact subsume its features, as well as providing for "intensive guidance on efficient drug development ... beginning as early as Phase 1 ," with specific recognition of advances in clinical trial design, such as adaptive clinical trials, as well as evolving technology, such as companion diagnostics. Most notably, the BTD offers the sponsor "organizational commitment involving senior managers," which has been likened to an "all hands on deck" call for collaborative, cross-disciplinary engagement by the FDA, not just at the division level but across all levels of management.

\subsubsection{Prioritization and Access to FRPs}

Cancer, AIDS, and orphan diseases were intentionally the focal point of the FDA's special programs in the 1980s and 1990s. However, as the public health emergency status of these diseases has been addressed to some degree in the 2000s, indeed AIDS and to some degree cancer have become "chronic diseases," others have surged to the forefront, including type 2 diabetes, depression, heart disease (especially in women), pandemic flu, and drug-resistant bacteria. For example, the threat level that AIDS represented in the 1980s and 1990s now confronts the USA in the 2000s in a different guise: flu pandemics, severe acute respiratory syndrome (SARS), and Clostridium difficile-associated diarrhea. For example, the cost to the US healthcare system of infections caused by antibiotic-resistant pathogens is $\$ 21$ to $\$ 34$ billion per year, with more than 8 million additional hospital days, whereas the medical costs of providing lifetime care for the 1.1 million people living with AIDS are \$20 billion per year.

How have the therapeutic areas that represent these diseases - anti-infective, central nervous system (CNS), CV, and metabolic/endocrinebeen served by the FDA's special designation programs in the 2000s? It is a very important question not only for the USA but for the world as well. Together with oncology, these four therapeutic areas comprise $75 \%$ of the pipeline in the USA from which $50 \%$ of global new active substances will flow, and they also encompass 13 of the 16 diseases and conditions identified by the World Health Organization as being global priorities for public health where there are pharmaceutical gaps. Yet, while oncology (and, to a lesser degree, HIV/AIDS) has been well served in the 2000 s, with $38-71 \%$ of the FDA's special designations (depending on which of the programs is being considered), the other four major therapeutic areas noted above have not benefited to anywhere near the same degree, with only 24$47 \%$ of FDA's designations being awarded to them.

\subsubsection{Emerging Sponsors and Paradigm Shift}

An analysis of drugs discontinued during development from 2001 to 2011 showed that financial and strategic factors were responsible for $56 \%$ of the discontinuations and safety, efficacy, and quality considerations for the remainder. This highlights an important change in the 2000s. In earlier years, it would have been anathema for the USFDA to take into consideration the impact of its programs on the investment community. In the wake of the new economic reality of limited resources for drug R\&D from both public and private sources, and the recognition that an increasing proportion of approved drugs are owned by venture-backed "emerging sponsors," that has all changed. An emerging sponsor is defined as the sponsor listed on the FDA approval letter who, at the time of approval, was not a holder of an approved application. Sponsors are still classified as "emerging" even if they have partnership or parent relationships with sponsors of a currently approved product. Of recent new molecular entity/new biological entity approvals approximately $40 \%$ belonged to emerging sponsors. We know that small companies are more likely to have multi-cycle 
Fig. 7.2 Fate of orphan product companies from 2007 to 2011
$\%$ of 2007 companies that

continued to be sponsors

in 2011

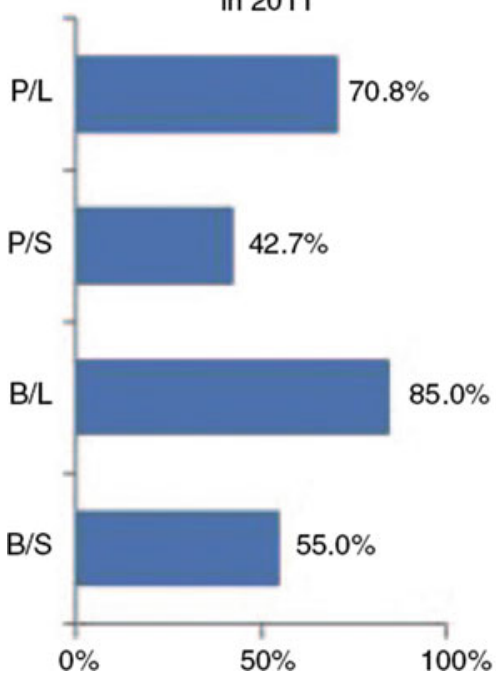

$\%$ of 2011 companies that are new sponsors

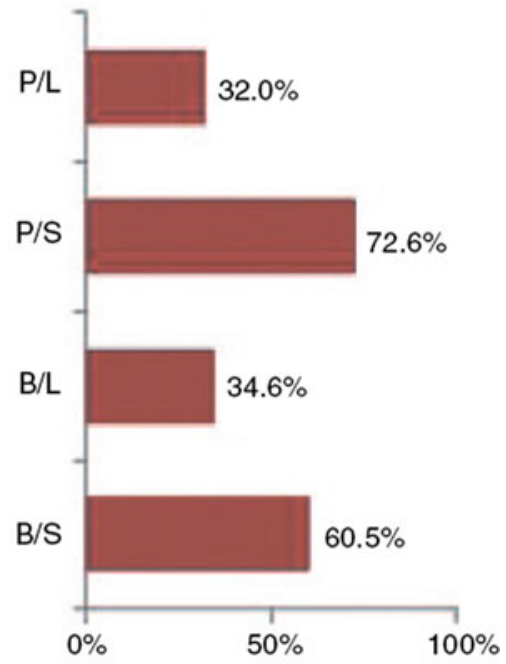

review, and less likely to garner approvals, with a $50 \%$ approval rate as compared with $80 \%$ for medium/large companies, according to an FDA study. Thus, emerging sponsors need more FDA assistance, and they benefit from a more structured process to ensure that discussion of prospects for special designation occurs early in the development program. A lack of predictability makes it difficult for sponsors to manage their portfolio and for small companies, in particular, to raise additional funds to bring those trials forward.

It has been shown in studies by the Tufts Center for the Study of Drug Development and others that priority review is important to investors, and consequently fast track is important because it is a harbinger of likely priority review and FDA flexibility on risk-benefit at a time closer to the "valley of death" (i.e., the time period from late discovery into early clinical development during which the flow of funds often dries up). During the congressional testimony on the Advancing Breakthrough Therapies for Patients Act, it was specifically noted that the legislation was supported in particular by the National Venture Capital Association. As with predecessor incentives, the real benefit of the BTD may be perceptual. One small company commenter said that the BTD may provide the certainty that investors want, whereas an investment commenter stated that the incentive structure is changing, moving away from incrementalism, and that breakthrough therapies are consistent with what insurers are looking for. In some critical areas, even in the face of daunting "push" hurdles, "pull" rewards can often be a sufficient incentive to sustain investment support, as novel antibiotics may now be considered an attractive opportunity due to the GAIN Act. According to a recent newsletter for investors, the new drug research and development paradigm shifted rapidly from traditional Big Pharma to venture capital-backed small companies, with emerging sponsors becoming increasingly crucial to the future of innovation, particularly in challenging areas of R\&D. Although smaller companies are often the seedbeds of new products and platforms for unmet medical needs, the example of orphan product $\mathrm{R} \& \mathrm{D}$ indicates that emerging sponsors come and go quickly, and much of their pipeline is at an early stage of development. 
For example, Fig. 7.2 details how dramatic a change orphan drug sponsorship experienced from 2007 to 2011, losing 150 companies that were in business at the time of the 2007 baseline accounting, but gaining $\sim 200$ new companies by 2011 . The greatest change occurred among smaller companies as pharma/small $(\mathrm{P} / \mathrm{S})$ and biotech/small (B/S) have considerably lower percentages of companies that remained "in the game" from 2007 to 2011, and yet comprised the lion's share of companies new to orphan product R\&D in 2011.

\subsubsection{Patient-Focused Drug Development (PFDD)}

Patient-focused drug development is an important new construct in the emerging paradigm for drug development. According to Theresa Mullin, the FDA's Center for Drug Evaluation and Research's Associate Director for Planning and Informatics, patient-focused drug development is a term used by the FDA in describing its efforts to ensure that the review process benefits from a systematic approach to obtaining patient perspectives on disease severity or unmet medical need. Expediting development for unmet medical needs will require a change in philosophy, one that can be undertaken only with the help of patient advocates themselves, in line with the new appreciation for patient-focused (also called patient-centered) drug development. The threshold for acceptable risk - the stumbling block for advancing HIV drugs decades ago-must again be re-evaluated and tailored to the willingness of patients to enable developers to make drugs (and of the FDA to review them) that have a narrower margin between risks and benefits. To this end, FDASIA allows: patients to participate in "appropriate agency meetings"; conflict-of-interest caps to be removed to make eligible a broader swath of stakeholders, such as patient advocates and consumer representatives; and pilot programs for patient participation to be put in place at the Center for Drug Evaluation and Research's divisions of oncology, gastroenterology, and antivirals; the Center for Devices and
Radiological Health's offices dealing with in vitro diagnostics and cardiac devices; and selected units within the Center for Devices and Radiological Health and the Center for Biologics Evaluation and Research.

Update and Commentary- $\mathrm{A}$ recent Tufts CSDD/DIA (Drug Information Association) study examined patient-centric activities implemented by pharmaceutical, biotechnology, and contract research organizations, as well as activities being piloted or in the planning stages. A global industry survey was conducted across pharmaceutical, biotechnology, and contract research organizations, assessing 25 patientcentric activities within clinical research. Some of these initiatives involve the use of social media to engage with patients, or the use of social listening to monitor study activity. Twenty-two unique companies responded to the survey, representing a mix of large, mid-sized, and small organizations. The most widely adopted patient-centric initiatives, including activities both implemented and piloted across organizations, were patient advisory boards (17 companies), professional panels (16 companies), lay-language clinical trial results summaries (13 companies), assessment of the patientorganization landscape (10 companies), and the use of home nursing networks (9 companies). The results suggest that organizations have a varied approach to the adoption and implementation of patient-centric initiatives, with more activities occurring in the planning stages than are being piloted or implemented. Many factors affect implementation and adoption, including buy-in by senior management, organizational vision, resources, and level of investment [8].

\subsection{What Happened to the MCM Sector from 2008 to 2016? [9]}

Beginning in 2008, the Tufts CSDD has routinely explored the R\&D landscape of Medical Countermeasures, which encompasses biologics, drugs, devices that may be used for biodefense against biological, chemical, and radiological bioweapons, or in the event of naturally 
(a)

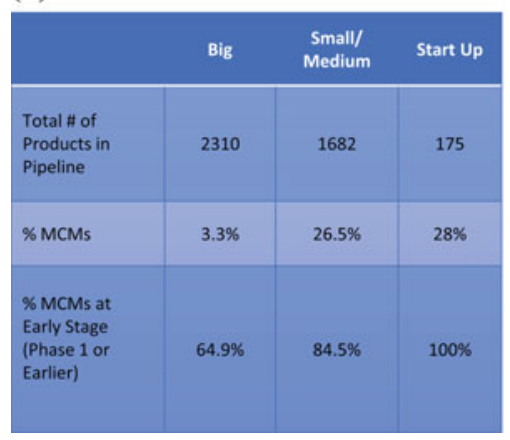

(b)

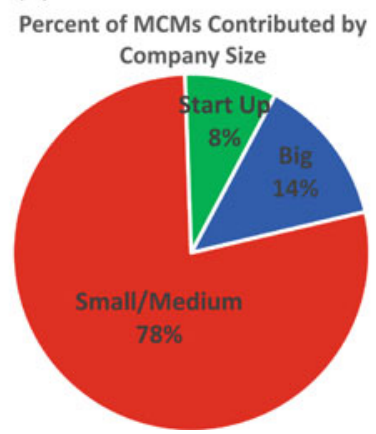

(c)

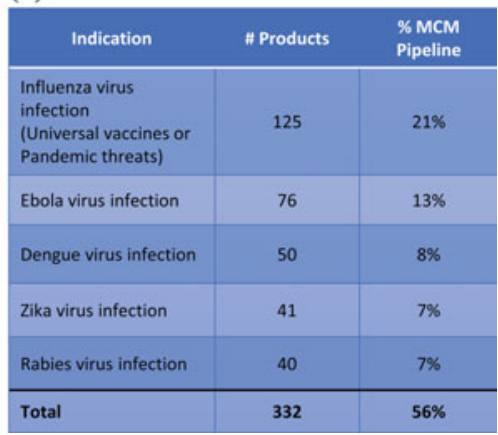

Fig. 7.3 MCM company, pipeline, and top 5 indications in 2016

occurring emerging and re-emerging diseases, or natural disasters. CSDD's most recent review (see Fig. 7.3) reveals that while some aspects of the field remain unchanged, there have been some significant changes as well. Broadly speaking, companies in the MCM field are typically privately owned, small to medium-sized enterprises (SMEs), with a biotechnology focus. While more than half of these companies are headquartered in the USA, there are now more non-US companies than in the past (48\%). China with 33 companies, the UK with 12, and Canada and Switzerland, both with 10 companies, together with the USA's 144 companies, round out the top five countries.

The most significant change in the landscape is the size of the MCM pipeline. In 2008, there were roughly 263 countermeasures in development. By 2016, that number had reached 592. Similarly, in 2008 there were around 133 companies working on MCMs. In 2016, there were 303. Continued, steady pipeline growth seems to indicate a positive impact from programs intended to encourage and support the development of MCMs, such as Project BioShield. It was established in 2004, with an initial budget of $\$ 5.6$ billion through FY2013, and since 2006 has been managed by the Biomedical Advanced Research \& Development Authority (BARDA), the overarching MCM authority within the U.S. Department of Health \& Human Services. BARDA and Project BioShield budgets have grown steadily, with budget increases in 2016 totaling more than $\$ 400$ mil
USD over their 2015 budgets, signaling to biopharma companies that MCM development is of continuing interest to the US government (USG). Much of the USG's support for SMEs comes in the form of Broad Agency Announcements (BAA) and Funding Opportunity Announcements (FOA), which allow smaller companies to compete for grants, awards, and contracts by conducting specified research projects.

This support for SMEs is vital as they are developing $78 \%$ of all MCM products currently in the pipeline. Looking at the numbers a little differently is also telling. Out of the 2310 total products in development by Big Pharma (i.e., top 25 biopharma companies), only $3.3 \%$ of them are MCMs. SMEs and start-ups, in contrast, have a much greater focus on the MCM arm of their portfolios at $26.5 \%$ and $28 \%$, respectively.

Another aspect of the Medical Countermeasure landscape worth examining is the relative role played by Big Pharma, SMEs, and start-ups in moving products from early development to later stages. Of the 592 products in development, $488(82 \%)$ are in early stages (Phase 1 or earlier), but among Big Pharma only $65 \%$ of MCMs are in early development, while among SMEs that figure rises to almost $85 \%$, and among start-ups the figure is $100 \%$ (almost by definition as start-ups are typically described as relatively new, small, privately-funded companies with no products on the market). Hence, Big Pharma is important for getting products through later stage development on the way to market, but the 

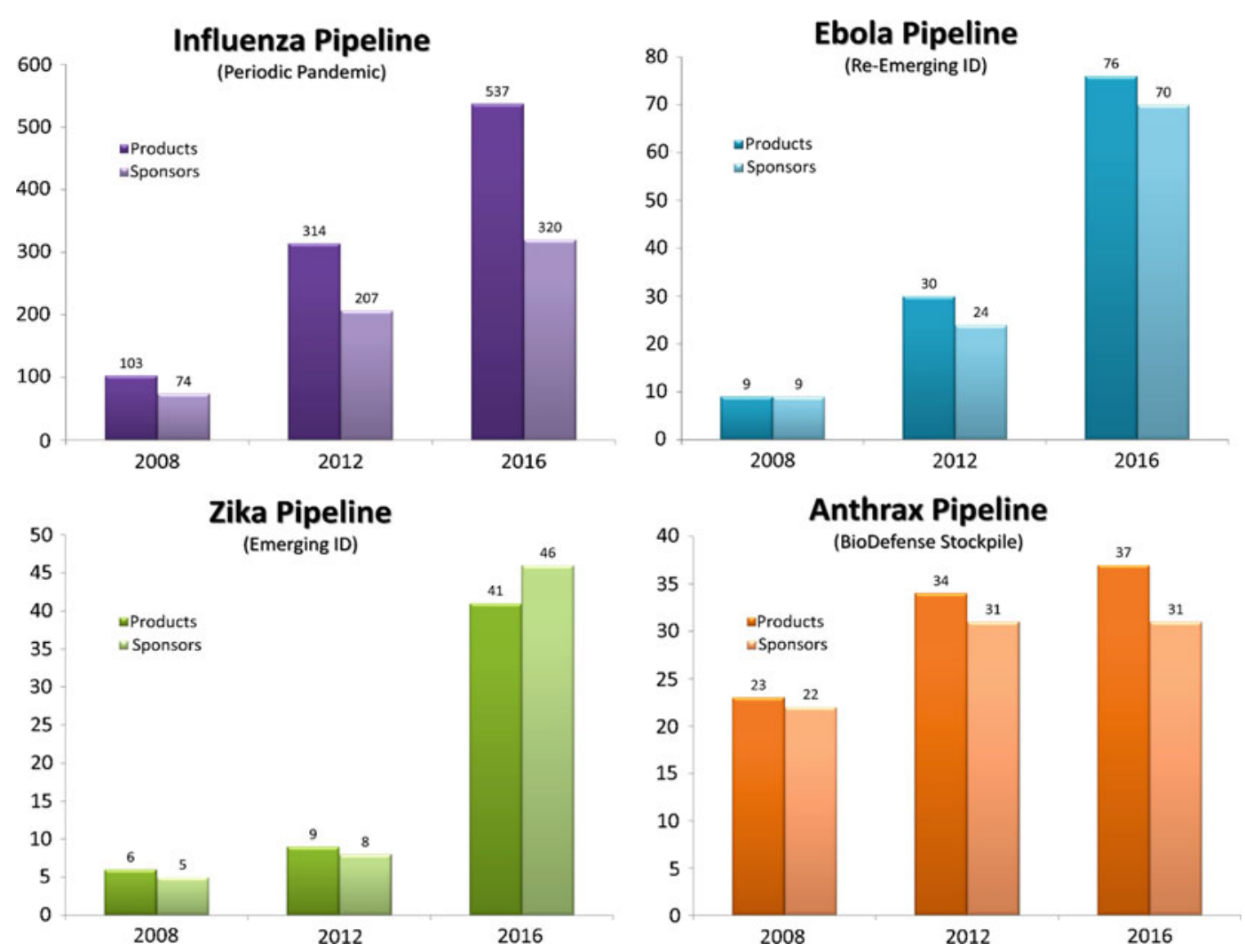

Fig. 7.4 Pipeline landscape snapshots for MCM category exemplars

seedbed for discovery to early development translation resides in SMEs and start-ups.

The five most prevalent indications in the MCMs pipeline provide some insight into a strong driving factor behind how a company decides the indications for which they will develop countermeasures. All five of the most common countermeasures have applications other than biodefense. Influenza MCMs are by far the most prevalent; there are 125 universal vaccines or vaccines for potential pandemic threats in production. These countermeasures alone make up $21 \%$ of the MCM pipeline. A broader look at all influenza products in development shows rapid growth from 103 products in 2008, to 314 in 2012, to 537 in 2016. Frequent influenza outbreaks make this rapid and sustained growth within the pipeline unsurprising. Periodic pandemics of various strains such as avian flu, swine flu, and $\mathrm{H} 1 \mathrm{~N} 1$ and their associated economic impacts have spurred interest in universal vaccines that protect against multiple strains of flu and avoid the need to respond to the current flu du jour by rushing to create a new vaccine.

Similar, though less dramatic, trends can be seen among other top countermeasures which have also seen recent outbreaks (see Fig. 7.4). Ebola countermeasures increased from 9 in 2008, to 30 in 2012, to 76 in 2016. Zika countermeasures increased from 6 in 2008, to 9 in 2012, to 41 in 2016. In 2014, Ebola had the largest outbreak in its 40-year history, and similarly in $2015 / 16$, there was the first large outbreak of Zika. These events demonstrated the urgent and unmet medical needs for treatment and prevention presented by emerging and re-emerging infectious diseases (ID) that are naturally occurring, sporadic, and non-biodefense, yet potentially profitable as future outbreaks of these or 
similar diseases are very likely. On the other hand, biodefense-only countermeasures tend to be purchased in bulk by governments and placed in readiness in something like the USG's Strategic National Stockpile. Ideally, such MCMs are rarely or never used, eventually reaching a target plateau in terms of "market" growth determined by the requirement to replace expired stock or expansion in the populations at-risk. Bacillus anthracis infection (Anthrax) illustrates this point very well. In 2008, there were 23 countermeasures being developed. In 2012 , there were 34 , and by 2016 that number had essentially plateaued at 37 .

Considering the fact that the top five indications on the list (influenza, Ebola, dengue fever, Zika, and rabies) comprise over half of all of the MCMs in development, it is clear that industry efforts are concentrated on a relatively narrow stream of the potential threat bandwidth. These five indications have a total of 332 products currently in development and average 66 countermeasures per indication. The remaining 57 indications, however, on the U.S. National Institute of Allergy and Infectious Diseases (NIAID) list of Emerging Infectious Diseases and Pathogens have a total of only 289 products currently in the pipeline, averaging 4.5 MCMs per indication (ranging from 0 to 37 per indication). Some very deadly diseases are in this group. Marburg virus, a virus related to Ebola, currently has only 12 countermeasures in development, 9 of which are still in discovery. Eastern Equine Encephalitis, which sees small but recurring outbreaks in the USA, and whose mortality rate of up to $75 \%$ makes it the deadliest mosquito-borne disease in North America, has only four MCMs in development.

Taken as a whole, it appears that while the budgets and prioritization schemes of government departments such as NIAID and BARDA affect the overall size of the Medical Countermeasure pipeline, it is current and recent world events - particularly in the form of emerging and re-emerging ID and pandemic outbreaks - that determine which countermeasures experience pulses in pipeline growth.

\subsection{Key Factors for the Future: Proving and Paying for Value [10]}

After surviving the "valley of death," the precarious period that exists between late discovery and early clinical trials, the next critical juncture for an innovative product is getting buy-in from USFDA to award it special status in one of its facilitated regulatory programs (FRPs), which expedite development and regulatory review. At that point, however, the imprimatur of the USFDA only goes so far to predict future success after launch. The path to commercial success can certainly be delayed by obstacles during the technical process of getting a product through the hurdles of proving safety, effectiveness, and product quality, but the last hurdle is always proving value - to physicians, patients, and especially third-party payers, both private and public. A study by Tufts CSDD in the early 2000s showed that technical success and commercial success do not always go hand-in-hand. That seminal study reported that of 15 major companies, there was a wide range of correlations between technical and commercial success. That gap has only become more challenging over time. Even though the time of development has remained somewhat static over the last decade, the cost of development has doubled, and overall success rates have declined. Thus, the ramifications of a market failure are much more daunting than in the previous decade, when only 3 out of 10 marketed products earned enough to pay their own freight (and generally the sunk costs of the other products on the market as well).

While the number of "Big Pharma" companies decreased by half in the 2000s, emerging sponsors are now responsible for close to half of novel approvals in the USA as well as ownership of $80 \%$ of the R\&D pipeline. For these small, start-up companies, reaching the market only to be thwarted by difficult reimbursement conditions or outright rejection for formulary inclusion can be disastrous for the company as a whole. Yet, these very companies are often where the seedbeds of innovation are most fertile, as mid-sized companies often have limited their 
portfolios to a few therapeutic areas and some larger companies over time have been abandoning certain therapeutic areas (such as CNS disease) after experiencing a lack of success or portfolio realignment due to merger and acquisition or new leadership.

On the commercial side what has to happen to balance the prospects of success for novel products generated by innovative platforms? Two concepts must become mainstream preceptspatient access schemes, more commonly referred to in the literature as risk-sharing agreements (RSAs), and real-world evidence (RWE). These concepts are intertwined and must be integrated to provide a solution for moving therapeutic options forward at the speed of science.

Risk-sharing entails agreements in which the buyer and seller believe that a product is sufficiently promising that it warrants the taking of certain risks by all parties because of the likelihood of potential benefits, i.e., value to the patient and thus to the healthcare system responsible for care and coverage, even if that benefit may be as barebones as "it's better than nothing." The first fundamental factor involved in risk-sharing is that all parties actually share the risk. This is where payers have often been found wanting, either by requesting too much proof too early (i.e., pre-approval) or by an unwillingness to accept any risk at all for an untried product without regard to regulatory approval or patient need. As counterproductive as this seems, there are many examples of this being the case with urgent medical needs in orphan drugs, personalized medicines, and most recently, abuse-deterrent formulations (ADFs) for opioids. In a 2014 study by Tufts CSDD of orphan drugs approved from 1983 to 2013, 9 out of 10 drugs had at least one condition restricting reimbursement, whereas for the 11 most expensive orphan drugs, patient cost-sharing ranged from 20 to $35 \%$ for drugs costing on average $\$ 400,000$ annually. For an early cohort of ten personalized medicines in 2013, Tufts CSDD found that payers reimbursed all drugs with variable and relatively high payer co-insurance and formulary restrictions, but reimbursement for the companion diagnostics was limited and highly variable.
By 2015, product developers still considered reimbursement to be a 4 out of 5 on an index of the most challenging factors facing personalized medicine. The problem is not confined just to private insurers. For example, the opioid abuse epidemic in the USA sounded the clarion call for ADF products. There has been a laudable response by manufacturers with 25-30 new applications pending review, 10 approved, and 4 launched by mid-2017, despite the fact that two years earlier $96 \%$ of prescribed opioids were not ADF products. Nonetheless, coverage by the US government under the Medicare program ranges from only 8 to $54 \%$ for these four critically needed products. ${ }^{10}$

Payers would say, in their defense, that they must see proof of clinical utility, i.e., that the drug, drug-diagnostic combination, or formulation demonstrate statistically robust evidence of positive outcomes for the patients for a sufficiently high proportion of patients. The problem is a Catch-22 (i.e., a difficult circumstance from which there is no escape because of mutually conflicting conditions). You cannot provide the quantum of evidence necessary for comparative effectiveness that payers demand until there are a sufficient number of patients who have experienced a therapeutic trial of the product in real-world settings. For this to happen, payers must assume certain risks a priori. It is a hard lesson. One that FDA has struggled to learn, but has finally become reconciled to it as a necessary regulatory paradox - accepting a certain amount of uncertainty in order to advance promising new technologies. Now payers have to "walk a mile" in the shoes of patients, care-givers, and regulators and take this same "leap of faith."

Arguably, faith is not a strong point among public or private payers, and probably we don't

\footnotetext{
${ }^{10}$ Opioid mismanagement is a worldwide problemalthough $80 \%$ of the global supply of opioids is currently consumed in the USA, it is a growing threat to global public health. In the EU, prescription opioids cause three-quarters of overdose deaths of teens and young adults, aged 15-39 years old. Often unheralded among the problems related to opioids is that half of all deaths that occur across the globe each year happen among people without access to pain medications.
} 
want it to be. We do want them to be able to make decisions on the best available evidence at the earliest point in time to meet an unmet need as soon as possible. This is where real-world evidence (RWE) comes into play. RWE is defined as data regarding the usage, or potential benefits or risks, of a drug derived from sources other than randomized controlled clinical trials, such as observational studies, registries, insurance claims databases, electronic medical records, wearable devices as well as patientcentered outcomes studies. The regulators are beginning to accept that RWE can be a telling source of evidence to assess the value over time in the life cycle of a marketed drug, and perhaps even answer questions that hadn't been asked yet, but should have been. However, there is some reticence to substitute RWE for randomized controlled trials (RCTs) as the gold standard for providing the necessary quantum of proof for initial approval, although it is gaining credence as a supplement to pivotal RCTs in this regard. In fact, FDA's premier regulatory science experiment - the Oncology Center of Excellence-now just a year old, works to incorporate RWE among just a handful of key advances in regulatory decision-making along with revamping trial design to eliminate the arbitrary phases $1,2,3$ structure, employing master protocols, re-defining trial eligibility criteria in conjunction with patient advocacy organizations to ensure representativeness, and reaching out to professional colleagues outside the agency as well as external stakeholders to generate better patient-reported outcomes metrics and instruments. Payers, for their part, have to shake off the shackles that bind them to a decision process that requires upfront proof of clinical superiority or rejection as the only two options for whether or not to reimburse a new drug (or a sliding scale of incremental cost-effectiveness that purports to implement a public health rationale of "greatest good for the greatest number of people" but is really thinly disguised rationing). Real-world evidence in its simplest terms is evidence from the patient, by the patient, for the patient. If the system is serious about becoming more patent-centric - as it should be since regulators serve the people, and patients are the end-customer for manufacturers and payersthen the experience of the broadest swath of patients in the widest range of practice settings should inform payer decisions. Who will pay and how is a different question, and depends on individual, familial, community, and nation-state support as well as cultural norms, but the first threshold is to establish whether a product has sufficient value to bring this next set of considerations into play.

Through a panoply of government, private, and individual payer systems, the ingredients exist to craft fair pricing and coverage solutions worldwide as much or even more rapidly than in the USA. Many nation-states in developed regions have nascent infrastructure or even burgeoning programs to provide for a system of real-world data collection that could provide an adequate source of RWE. At the same time, RSA models are not confined to any one global region but are ongoing initiatives in many countries (including in China and South Korea according to a recent ISPOR workshop summary). With the judicious implementation of RSAs and RWE, access to novel medicines and a conducive environment for innovative technology can be a reality on the short-term horizon not just for a few countries but many emerging markets, and from there the rest of the world.

\subsection{Global NAS Launches 2013-2017: Trends and Implications [11]}

The term new active substances (NASs) originated with the European Medicines Agency (EMA) and is defined as a new product that contains an active substance that was not previously authorized, is not related to any other previously authorized substances, and differs significantly in safety and/or efficacy. NAS launches serve as a surrogate measure for evaluating innovation trends in chemical and biological discovery and development in addition to patent and clinical trial data and generally in place of other forms of sourcing data such as the 


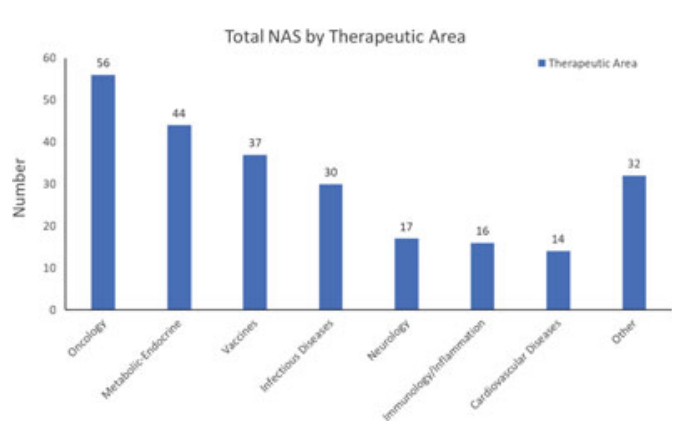

Fig. 7.5 Global NAS launches by therapeutic area 2013-2017

country in which the sponsor company is headquartered because globalization of the industry and the marketplace have rendered such data unreliable for such evaluations. NAS launch data is a reliable indicator of the sponsor's target country and indication because nearly half of all new drugs are launched in ten or fewer countries, often with long lags from first launch to subsequent launches. ${ }^{11}$

NAS output during 2013-2017 was quite variable, ranging from 40 to 60 , and averaging 49 , a yet substantial increase over the average for the twenty-oughts at 32 and even the teens overall at 46. The current cornucopia of NAS reflects the favorable economic environment as well as the expansive number of companies and compounds in the field, over 4000 companies and over 15,000 drugs in the pipeline. The US share of first launches worldwide remains dominant at 60-65\% during 2013-2017, having increased steadily from a low point of $45 \%$ in 2010 according to FDA. Nonetheless, Asia is a fast comer. As shown in Fig. 7.5, the NAS output of Japan alone (30) is equal to the entire output of the EU (33). And when the output of Japan is added to that of the rest of the Asia-Pacific region, it is nearly half that of the USA (65 vs. 74/148, respectively).

Oncology currently makes up $34 \%$ of the global industry pipeline and $23 \%$ of NAS from 2013 to 2017 . Although the metabolic-endocrine

\footnotetext{
${ }^{11}$ Cockburn, I. et al. Patents and the Global Diffusion of New Drugs. American Economic Review 2016, available at http://eprints.1se.ac.uk/65415/.
}

category appears in this Figure to be the second most common therapeutic area for NASs, it is really a composite category comprised of drugs for endocrine diseases such as type 2 diabetes and a broad array of metabolic drugs for congenital enzyme deficiencies (i.e., orphan drugs for rare conditions). Infectious disease represented by both columns 3 and 4 in Fig. 7.5 is the second most common therapeutic area among NAS - a positive trend. However, the fact that neurological disease (including Parkinson's and Alzheimer's diseases) and cardiovascular diseases trail oncology by a considerable margin is a negative trend and brings into question the prioritization agenda of both the private and public sectors. During 2013-2017, oncology, diabetes, and orphan drugs comprised from 37 to $54 \%$ of the NAS launched worldwide, indicating that nearly half $(46 \%)$ of all new drug approvals worldwide addressed a limited set of the most significant threats to global public health. For example, according to the WHO Global Burden of Disease study published in 2012, they recorded the following Global Death Ranks respectively in 1990 and 2010:

\begin{tabular}{l|l|l}
\hline Ischemic heart disease & 1 st & 1st \\
\hline Stroke & 2nd & 2nd \\
\hline Respiratory disease & 3rd & 4 th \\
\hline Top four cancers & 8-24th & $5-19$ th \\
\hline
\end{tabular}

The USA produces the majority of NAS overall at around $60 \%$, but the vast majority of oncology drugs at $82 \%$ and infectious disease

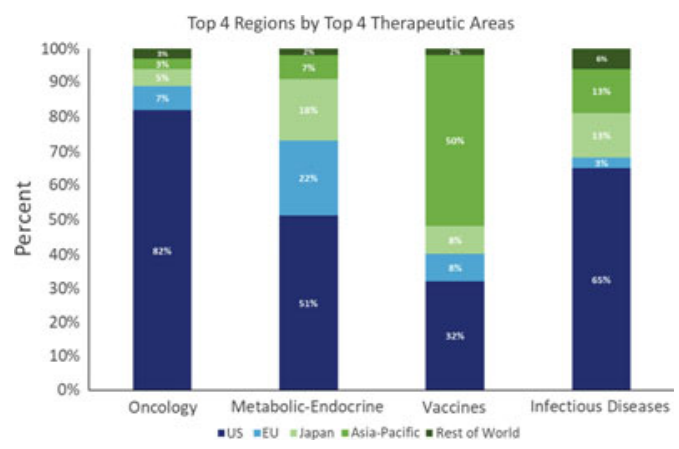

Fig. 7.6 Global NAS launches by region and ta 20132017 
treatments at $65 \%$ (see Fig. 7.6). The metabolicendocrine field is more evenly divided among the USA (at just over 50\%), Europe and Japan (at about 20\% each), with Japan emphasizing endocrine drugs for diabetes type 2 and the EU focusing on enzyme replacement treatments for orphan conditions.

Significantly, the Asia-Pacific region, in particular China and India, are the major sources of vaccines for the worldwide market at $50 \%$. In sum, the report highlights that while the USA remains dominant as the source of NAS and oncology remains the dominant therapeutic area, change is coming. We see evidence of such changes, the challenge of infectious disease to the hegemony of the oncology therapeutic area, as well as the new role of Asia-Pacific emerging market powers in $\mathrm{R} \& \mathrm{D}$ and the upsurge of the metabolic-endocrine therapeutic area worldwide due to new treatments for diabetes and rare diseases.

\subsection{What the MCM Experience Portends for Drug Development in 2020 and Beyond}

Taking an overarching perspective at what happened in the years encompassed by our review of the MCM experience reveals how the industry evolved and de-evolved in response to a web of economic, political, and public health events. Economically, specialty markets such as targeted cancer drugs and orphan drugs are outcompeting generalist disease markets like CVD, respiratory, and GI because investment dollars respond to the new shibboleth of "personalized medicine," and innovative approaches for serious disease are better-received by private payers and meeting unmet medical needs by public payers. Diabetes is beating the trend inveighing against the generalist market because it is widespread, well-covered, and cost-effective. Infectious disease therapeutics are currently beating the headwinds of the past because of the push from low comparative $R \& D$ costs along with high success rates due to recent regulatory incentives.
Meanwhile, emerging markets' countries in the Asia-Pacific region are picking up the slack in vaccines by building on their capital investment in manufacturing equipment and facilities and institutional expertise in generics production.

Politically, industry and the public health community realized by this time the power that patient advocacy through groups such as the American Cancer Society and NORD (National Organization for Rare Diseases) wielded and the impact they had had in a few short decades to focus not only government but industry priorities. This generated a public health lobby from a consortium of 50 or so mostly public health and employer groups that successfully stumped for the GAIN Act with dramatic results. So, what changed over time from the twenty-oughts to the twenty-teens was a very real series of pandemic threats from Swine flu and Bird flu in 2009, H1N1, Ebola, antimicrobial resistance, etc., and a diminishing of bioterror concerns, which became subsumed into a background impetus for MCM prioritization. Coincident with this and in part because of it, MCMs devolved from an emerging stand-alone biodefense sub-sector to merge instead into the more generalized expansion of infectious diseases R\&D for therapeutics, diagnostics, and preventatives.

In terms of public health, there is a growing realization that HIV/AIDS and many forms of cancer are now "chronic diseases" and not the death knell that once sounded such alarm among both the public and private sectors. While cancer is still a major priority, heightened attention to visible threats to the public health such as diabetes among an increasingly "older" population and the vulnerability of the general population to pandemics in the "global village" have begun to swing the pendulum back toward a focus on the unmet needs of the broader population. The challenge is that the "quick fix" of regulatory incentives is only a partial fix as it affects only the middle third of a typical drug's several-decade life cycle. It is certainly useful to shorten development and approval time on average from approximately 10 years to 7 years for prioritized products, but those 7 years are preceded by $7-10$ years in discovery and 
followed by 7-10 years of marketing. FRPs are better than nothing, but oncology has a competitive advantage in corporate decision-making for resource allotment because it benefits from decades of previous basic research that have created an expansive and expanding knowledge base on which to build future directions and reassess current ones. Diabetes on the other hand derives its favorable competitive position versus other therapeutic areas in the post-launch period because of the familiarity among providers and prescribers with the basics of the disease and a rising prevalence (fast approaching the certainty of death and taxes), as well as the pharma industry ROI "comfort level" of addressing a long-term chronic disease affected by both metabolic and behavioral factors.

What has to change to enhance our prospects for a future not driven by commerce and caprice, but foresight and forbearance? We have been faced with rumblings for a long time that something is going to "rock our world." These premonitory tremors have been cataclysmic events whose impact was limited or long ago, but now loom again as the primary threat to the future of civilization, if not, humankind's continued dominance of its environment. There are, however, some who believe that we cannot continue to depend on the defenses of time, distance....and luck forever, or even for the foreseeable future. Just consider how long mankind has been dealing with the nemesis of pain - the primary antagonist of quality of life with only variable success. And even now that success is threatened anew by the epidemic of opioid addiction, now being recognized as a double-edged Sword of Damocles - a threat to the teens and young adults of the developed world due to addiction and overdosing while fast-becoming a threat to the developing world as control measures and liability concerns exacerbate an already dire lack of access to pain medications.

Concern and concerted effort for our health and prosperity is considered to be the responsibility of our government as well as each individual. Yet, our responses to threats that are novel or widespread so far have been too little, too late. We can no longer afford such nonchalance. We must recognize that we are faced with a growing panoply of threats to public health - some at least of an unknown nature, size, and imminence. We know there are significant unmet medical needs, but we don't know exactly where they are and when they will reach critical mass...or the "point of no return." We do know, however, that if we don't begin to hammer together a network of fixed and flexible countermeasures, our only defenses will be draconian public health programs such as triage and quarantine for infectious disease (or rationing of pain medications in a mostly symbolic attempt to control addiction).

We have another choice. We can work on products to identify, treat, and prevent the harmful agents that we know, as well as accelerate and expand our capacity to identify and combat the ones we don't know. It will, however, require much more commitment, coordination, funding, and accountability than we have shown so far. This sounds easy in concept, but the reality is hard. There is the vexing problem of the tension between the long-term need for MCMs and the short-term horizon of our political systems. The likelihood of an event happening within any one person's working (or voting) lifetime is low, but the likelihood that the consequences of such an event would be extremely unpleasant is high. It is a basic paradox inherent in public health preparedness. If you are really good at it...nothing bad happens! But then complacency and second-guessing seep in and weaken the resolve and resource commitment. The way out of this conundrum is to put people in charge who have a clear vision of what needs to be done and provide them the independent authority, resources, and infrastructure to do it right the first time. We do not know when the time may come that we will not have a second chance to avoid cataclysmic casualties from a contagious super-spreader like pandemic flu or to play catch-up in a race with an insidious dealer of death like opioid mismanagement that should never have been a contest at all! The ancient Greeks framed the challenge eloquently and ineluctably: "A society grows wise only when old men plant trees under whose shade they will never sit!" 


\section{References}

1. Whalen J. Hurdles multiply for latest drugs. Wall Street J 1 August 2011.

2. Deloitte Consulting, 2018 report, "Unlocking R\&D Productivity: Measuring the return from pharmaceutical innovation 2018," available at www2.deloitte. com. Last accessed 6 Jan 2019.

3. U.S. Centers for Medicare \& Medicaid (CMS) report, National Health Expenditures 2017 (Highlights), available at www.cms.gov. Last accessed 6 Jan 2019.

4. Milne, C.-P. Is there a business in medical countermeasures for bioterror and pandemics? (White Paper, 2010). Tufts Center for the Study of Drug Development (Tufts CSDD); Boston, MA, USA.

5. Alex Murtha, Homeland Prep News, Friday, September 1, 2017. http://webcache.googleusercontent.com/ search?q=cache:YtWOf3fpbkgJ, https://homelandpre pnews.com/stories/24000-battelles-coleman-small-com panies-key-new-cbrn-medical-countermeasures/\&hl= en $\& g l=u s \&$ strip $=1 \& v w s r c=0$. Last accessed 26 Nov 2018.

6. Industry Experts Fall 2018, Global Influenza MarketVaccines and Therapeutics (Report Code PH015) at industry-experts.com. Last accessed January 6, 2019.
7. Milne C-P Clinical Pharmacology \& Therapeutics 2013;95(1):98-109 advance online publication 30 October 2013. https://doi.org/10.1038/clpt.2013.155.

8. Lamberti MJ, Awatin J. Mapping the landscape of patient-centric activities within clinical research. Clin Ther 39(11):2196-2202. https://doi.org/10.1016/j. clinthera.2017.09.010. Epub 2017 Oct 9. https:// www.ncbi.nlm.nih.gov/pubmed/29032849. Last accessed 26 Nov 2018.

9. Milne C-P, Smith ZP, Chakravarthy R. Landscape for medical countermeasure development. Nat Rev Drug Devel 2017; 16:448-449.

10. Milne C-P. Reaching a New Innovation Threshold. Pharma Focus Asia (editorial), Ochre Media Pvt. Ltd., Issue 30, 2018. Available at https://www.pharma focusasia.com/archives-magazine/81. Last accessed 26 Nov 2018.

11. Kaitin KI, ed. U.S. continues to lead new drug R\&D, but Asia-Pacific is starting to rise. Tufts CSDD Impact Report (January/February 2019), vol. 21, number 1. Tufts University School of Medicine, Center for the Study of Drug Development, Boston, MA, USA, 2019. 